\title{
THESIS
}

\section{THE EFFECTS OF MATERNAL RESPIRATORY SINUS ARRHYTHMIA AND BEHAVIORAL ENGAGEMENT ON MOTHER-CHILD PHYSIOLOGICAL COREGULATION}

\author{
Submitted by \\ Amanda M. Skoranski \\ Department of Human Development and Family Studies
}

In partial fulfillment of the requirements

For the Degree of Master of Science

Colorado State University

Fort Collins, Colorado

Summer 2017

Master's Committee:

Advisor: Erika Lunkenheimer

Rachel Lucas-Thompson

Renzo Cavalieri 
Copyright by Amanda Marie Skoranski 2017

All Rights Reserved 


\begin{abstract}
THE EFFECTS OF MATERNAL RESPIRATORY SINUS ARRHYTHMIA AND BEHAVIORAL ENGAGEMENT ON MOTHER-CHILD PHYSIOLOGICAL COREGULATION
\end{abstract}

Parent-child coregulation, observed as the active organization and coordination of parents' and children's behavioral and physiological states, is an important precursor for children's developing self-regulation, but we know little about how individual parent factors shape parent-child coregulation. We examined whether differences in maternal physiology and behavioral engagement were associated with coregulation of mothers' and their 3-year-old children's physiological states over time. We examined coregulation in real time by modeling maternal and child respiratory sinus arrhythmia (RSA) for 47 families across 18 minutes of dyadic interaction using multilevel coupled autoregressive models fitted in Mplus. Maternal basal RSA, maternal teaching, and maternal behavioral disengagement were each entered as between-subjects predictors to determine the extent to which mother-child coregulation was strengthened or weakened by maternal factors. Whereas greater maternal teaching during the mother-child interaction was associated with stronger coregulation in mother and child RSA over time, maternal disengagement was related to weaker coregulation: specifically, there were moredivergent parent and child RSA at higher levels of maternal disengagement. Coregulation in mother-child RSA was also weakened when mothers' basal RSA was higher. Findings contribute to the emerging knowledge base on real-time patterns of parent-child coregulation and 
suggest a role for parent-child physiological coregulation as a mechanism by which parent factors support or hinder children's developing self-regulation. 


\section{TABLE OF CONTENTS}

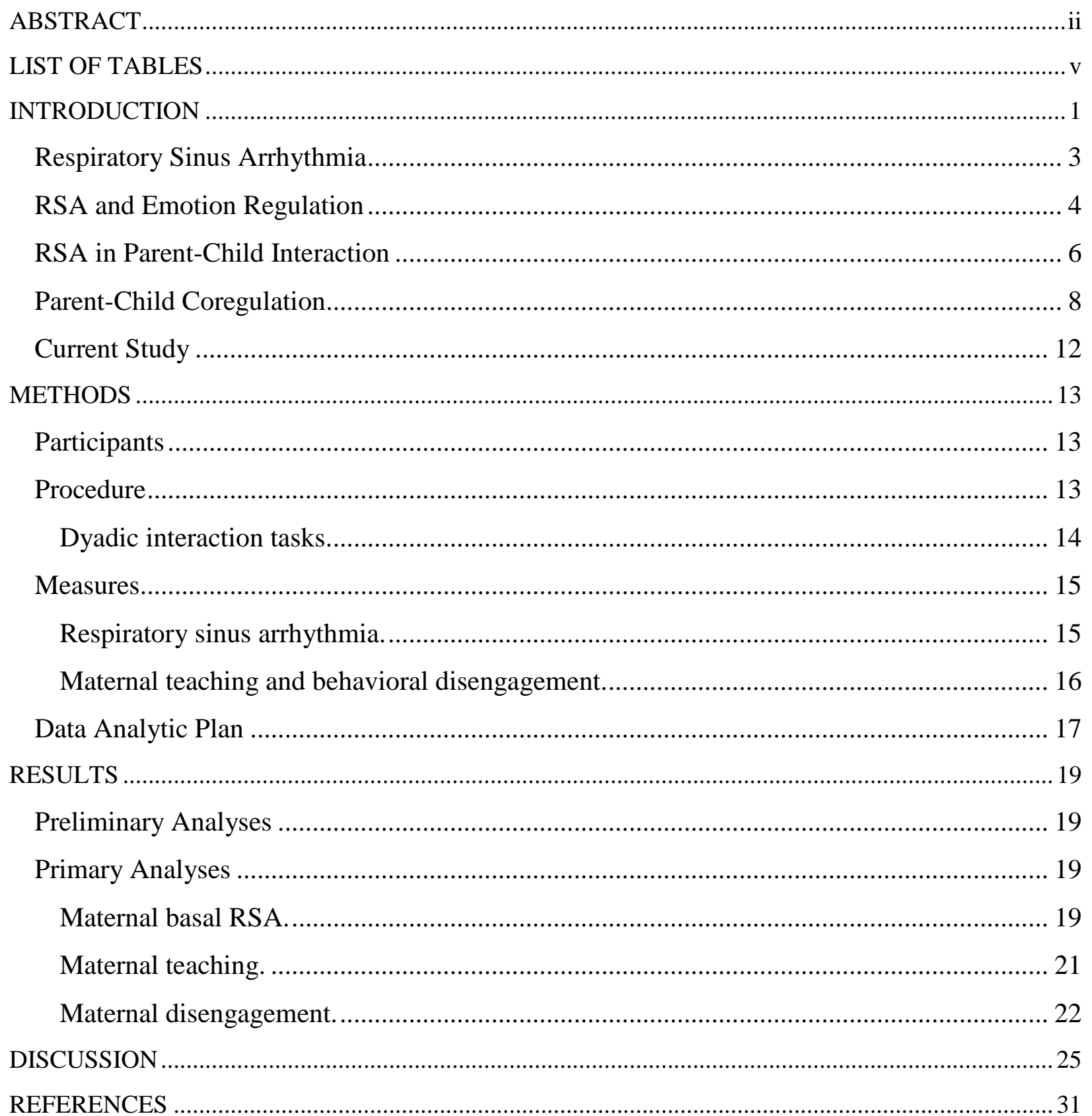




\section{LIST OF TABLES}

TABLE 1. MULTILEVEL COUPLED AUTORGRESSIVE MODELS PREDICTING CURRENT RSA FOR MOTHERS AND CHILDREN WITH MATERNAL BASAL RSA AS

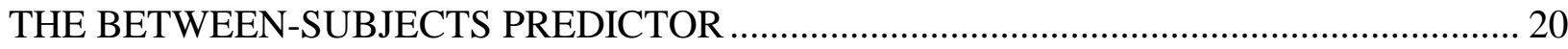

TABLE 2. MULTILEVEL COUPLED AUTOREGRESSIVE MODELS PREDICTING CURRENT RSA FOR MOTHERS AND CHILDREN WITH MATERNAL TEACHING AS THE BETWEEN-SUBJECTS PREDICTOR

TABLE 3. MULTILEVEL COUPLE AUTOREGRESSIVE MODELS PREDICTING CURRENT RSA FOR MOTHERS AND CHILDREN WITH MATERNAL DISENGAGEMENT AS THE BETWEEN-SUBJECTS PREDICTOR 


\section{INTRODUCTION}

The regulation of behavioral, emotional, and physiological processes is essential for adaptive functioning in human development. Self-regulation in early childhood is both a critical marker of adaptive functioning as well as a precursor for future competence across the lifespan, including cognitive proficiency, social functioning, and academic success (Diamond \& Aspinwall, 2003; Posner \& Rothbart, 2000). Regulatory processes come online early in development, however, capacity for self-regulation is limited in infancy and early childhood. Young children rely heavily on scaffolding processes from mutually-regulating interactions with trusted adults in order to regulate themselves (Tronick, 1989). Such mutually-regulating interactions in close interpersonal relationships provide support for neural development during a child's first few years of life, particularly with regard to social and emotional functioning (Siegel, 2001). Arguably one of the closest relationships for children throughout their lives occurs with their parents, with both parent and child growing and changing together through patterns of transactional exchanges (Sameroff \& MacKenzie, 2003). Thus, the parent-child relationship is an especially important context through which children learn to regulate both their physiological arousal and expressed emotions and behaviors early in life (Calkins, 2011).

One critical mechanism supporting child regulatory processes is parent-child coregulation, the active organization and coordination of parents' and children's behavior, emotions, and physiological states over time (Olson \& Lunkenheimer, 2009). The pattern of coregulation in parent-child interactions may be just as important as the content and quality of interaction in promoting child outcomes. When there is greater synchrony in parents' and children's emotional and behavioral expressions and physiological states (in other words, they 
tend to co-occur in real time), children's own self-regulatory abilities are promoted (Calkins, 2011; Feldman, 2012). For example, greater matching of positive affect between parents and preschool-aged children during parent-child interaction has been shown to contribute to the reduction of dysregulated behavior problems among children above and beyond children's positive affect alone (Lunkenheimer, Olson, Hollenstein, Sameroff, \& Winter, 2011).

Parent-child coregulation and children's individual attempts at self-regulation are inherently dynamic constructs that develop and unfold across multiple time scales (Olson \& Lunkenheimer, 2009). Patterns of exchanges between parents and children that unfold over a matter of minutes may have an impact on children's long-term development (Sameroff \& MacKenzie, 2003). The development of self-regulatory competence also involves multilevel coordination between physiological systems, affective and behavioral expression, and interpersonal processes (Siegel, 2001). In order to better understand the processes through which parent-child coregulation impacts the development of individual self-regulatory mechanisms in children, more research examining fine-grained temporal dynamics and regulation across physiological and behavioral levels of parent-child interaction is crucial (Cole, Martin, \& Dennis, 2004). The current study adds to this literature by examining coregulatory dynamics between parents and their 31/2 year-old children in real time (i.e., second-by-second) throughout the course of an 18-minute observed laboratory session. Given that parents should hold significant influence over their young children when guiding them to complete a task, we examined whether individual parent factors, specifically maternal physiology and behavioral engagement, predicted the dynamics of parent-child physiological coregulation. 


\section{Respiratory Sinus Arrhythmia}

Both self-regulation and coregulation can be assessed by examining underlying physiological processes, in addition to behavioral and emotional components (Porges, DoussardRoosevelt, \& Maita, 1994). Much research on physiological self-regulation in childhood has focused on the autonomic nervous system (ANS), which connects the brain to the body through afferent and efferent pathways, and serves as a mechanism for direct and quick regulation of bodily function based on neural input (Berntson, Cacioppo, \& Quigley, 1993). The two branches of the ANS, the sympathetic nervous system (SNS) and parasympathetic nervous system (PNS), serve distinct but interrelated functions. Whereas the SNS becomes active during times of stress, the PNS works to regulate the body's function and maintain homeostasis at times of rest (Beauchaine, 2001).

One common measure of physiological regulation is respiratory sinus arrhythmia (RSA), or the amount of variability in heart rate associated with the rate of respiration. Research has demonstrated that RSA is largely determined by outflow from the vagal nerve, which is a part of the PNS and functions as a "vagal brake" on the heart, slowing heart rate while an individual is at rest (Berntson et al., 1993). The level of RSA during these relatively low-stress periods, which we refer to as basal RSA, seems to reflect the ability for an individual to engage with the outside world (Beauchaine, 2001). In particular, higher basal RSA appears to facilitate an individual's capacity to participate in healthy social interactions (Porges, 2007). This may be due to the fact that higher basal RSA seems to reflect greater emotional range and regulation, which is demonstrated as greater expression of both positive and negative emotion in infancy, greater socioemotional competence in childhood, and reduced incidence of psychopathology, including depression, anxiety, and aggressive behaviors, in adolescence and adulthood 
(Beauchaine, 2001). Stability in basal RSA has been demonstrated early in development from 9 months to 3 years of age (Porges, Doussard-Roosevelt, Portales, \& Suess, 1994) and across the preschool years (Doussard-Roosevelt, Montgomery, \& Porges, 2003).

Self-regulation has also been assessed empirically by measuring the change in RSA during periods of heightened stress. When an individual experiences stress or challenge, the "vagal brake" is lifted and heart rate accelerates and RSA decreases (or withdraws). This process may reflect the adaptive reallocation of resources to the SNS, which helps mobilize the individual to respond to challenge (Beauchaine, 2001; Gunnar, Porter, Wolf, Rigatuso, \& Larson, 1995; Wilson \& Gottman, 1996). Thus, greater RSA withdrawal during stressful or challenging situations reflects more-effective physiological regulation (Porges, Doussard-Roosevelt, Portales, \& Greenspan, 1996). Conversely, increases in RSA to stress, or maintaining consistent levels of RSA throughout stressful encounters may reflect less efficient physiological regulation, or may be due in part to lower behavioral engagement or perceived distress in response to the emotional or cognitive demands of an encounter (Porges, 2007).

\section{RSA and Emotion Regulation}

Porges' Polyvagal Theory proposes that vagal tone, often measured as basal or resting RSA, is part of a complex physiological system that supports healthy social interaction (Porges, 2003). The vagal nerve has both afferent and efferent pathways that link with neural regions involved in both the expression and regulation of emotion (Berntson et al., 1993; Porges, 2003). It has been suggested that efficient regulation of the "vagal brake," reflected in levels of basal RSA and RSA withdrawal to stress, provides a crucial underlying support system for emotional expressivity and regulation, as well as social approach, which together facilitate adaptive interpersonal interactions (Porges, 1991, 2003, 2007). 
Empirical evidence supports this claim, as both basal RSA and RSA withdrawal during stress have been linked with emotional regulation and adaptive social behavior beginning early in development. Infants with high basal RSA exhibited less negative affect, and infants with greater RSA withdrawal to stress were rated by their mothers as more easily soothed (Huffman et al., 1998). Similarly, higher basal RSA was related to greater emotional reactivity to both positive and negative events (Fox, 1989), and greater RSA withdrawal was linked with greater social approach in infants (Stifter \& Corey, 2001). By contrast, poor RSA withdrawal to stress in infancy was predictive of increased behavior problems (Dale et al., 2011) and fear of strangers (Brooker et al., 2013) when children reach preschool age.

In early childhood, RSA withdrawal to an anger-induction task followed by return to a high basal level of RSA has been linked with more adaptive regulation of aggression (Miller et al., 2013). Relatedly, high basal RSA in 4-year-old children was associated with a decreasing trend in mother-rated negativity across the span of the next three years (Blandon, Calkins, Keane, \& O'Brien, 2010). In addition, greater RSA withdrawal to stress in infancy predicted better social skills for children once they entered preschool (Graziano, Keane, \& Calkins, 2007). Conversely, toddlers who showed less RSA withdrawal during cognitive and emotional challenges also showed greater negative affect and less regulatory behavior (Calkins \& Dedmon, 2000). Dysregulation of RSA in infants, toddlers, and preschool-aged children has also been linked to greater incidence of behavior problems (Calkins \& Dedmon, 2000; Calkins, Graziano, \& Keane, 2007; Calkins \& Keane, 2004) and aggression (Porges et al., 1996).

RSA continues to be an important factor in emotional health throughout the lifespan. Impairments in RSA withdrawal, particularly when in conjunction with low basal RSA, have been found to be associated with both internalizing and externalizing problems throughout 
childhood and adolescence (Graziano \& Derefinko, 2013). Similarly, adults with depression and/or anxiety symptoms tend to have lower basal RSA and show less RSA withdrawal in response to stress (Kemp et al., 2010; Kircanski, Waugh, Camacho, \& Gotlib, 2016). Taken together, evidence suggests that regulation of RSA is an important factor in the emergence of emotion regulation capacities during key developmental periods, which facilitates social engagement.

\section{RSA in Parent-Child Interaction}

Prior research has shown that parents can provide support for children's developing physiological regulation systems through positive behavioral engagement and responsiveness, particularly during stressful interactions. In general, children show improved RSA regulation (i.e., withdrawal of RSA during challenge and return to a high basal RSA during relatively lowstress periods) during collaborative tasks with a parent versus tasks completed on their own (Calkins, Graziano, Berdan, Keane, \& Degnan, 2008), which may reflect improved physiological regulation when children and parents work together. Mothers who show a high level of sensitivity and responsiveness to infants' needs tend to have children who show better regulation in the form of greater RSA withdrawal during an interactional task designed to elicit distress (Moore et al., 2009). Highly sensitive mothers also have infants who are better able to return to a higher level of basal RSA following a stressful encounter with a parent (Conradt \& Ablow, 2010). In contrast, mothers who engage in lower levels of socialization and touch tend to have infants with lower basal RSA (Feldman \& Eidelman, 2007). In addition, negative parenting (e.g., scolding, derogatory comments, controlling child's behavior) is associated with lower levels of RSA withdrawal in children during an emotional challenge (Calkins, Smith, Gill, \& Johnson, 1998), and poor parent-child relationship quality also predicts lower levels of RSA 
withdrawal in toddlers (Calkins et al., 2008). The relationship between parenting and RSA regulation in children may be reciprocal, for example, low withdrawal of RSA to stress in toddlers was shown to predict higher levels of negative parenting two years later (Kennedy, Rubin, Hastings, \& Maisel, 2004).

Positive parenting behavior may be supported by parents' own physiological regulation, including their level of basal RSA and their degree of RSA withdrawal during stressful encounters. The relationship between RSA and parenting behavior is evidenced by the fact that dysregulation of RSA in parents tends to be associated with fewer positive engagement behaviors with children. Mothers who have elevated cortisol stress responses (i.e., increased activity of the SNS) but poor RSA withdrawal to stress are more likely to engage in intrusive parenting behaviors with their infants (Mills-Koonce et al., 2009). Similarly, mothers who exhibit higher skin conductance (another index of SNS activation) but lower RSA withdrawal during a stressful parent-child interaction also tend to use fewer soothing behaviors with their infants (Ham \& Tronick, 2006). Furthermore, mothers who show less RSA withdrawal when attempting to soothe their infants following a period of separation are more likely to have infants with insecure attachment patterns (Hill-Soderlund et al., 2008). Mothers with elevated depressive symptoms tend to show poor RSA withdrawal in response to infant distress (Oppenheimer, Measelle, Laurent, \& Ablow, 2013), which may reflect less personal distress or less engagement in active coping in this stressful encounter (Porges, 2007). Altogether, this evidence may suggest that parents who do not show efficient RSA regulation during stressful interactions may also engage in fewer positive engagement behaviors with children, which in turn may be associated with dysregulated behavioral patterns in children. 


\section{Parent-Child Coregulation}

One mechanism through which parents' behavioral and physiological states may influence children's developing self-regulatory behavior is through alterations in coregulatory patterns during parent-child interactions. Synchrony, or time-dependent coordination, in parent's and children's behavior and physiology is an adaptive coregulatory pattern that develops across early infancy (Feldman, 2007). Over time, mothers and children become sensitized to one another's behavioral and physiological cues, enabling greater coordination of parent-child states (Feldman, 2012). Prior research suggests that physiological synchrony between parents and children is normative (Lunkenheimer, Tiberio, Buss, Boker, \& Timpe, 2015). For example, mothers' and children's thermal signatures, a measure of autonomic function, are synchronous while mothers watch their children perform emotionally challenging tasks (Ebisch et al., 2012; Manini et al., 2013). Synchrony in physiological states has been linked to greater behavioral coordination between parents and children; for example, mothers and infants tend to show greater coordination in heart rate during periods of behavioral synchrony, such as gaze sharing (Feldman, Magori-Cohen, Galili, Singer, \& Louzoun, 2011).

Synchrony in parent-child physiological and behavioral coregulation may be an important precursor for children's self-regulatory behavior and social competence (Feldman, 2007). Synchronous coregulation of emotion between parents and children, when paired with a high level of positive emotion, has been found to predict lower behavioral dysregulation in children (Lunkenheimer et al., 2011). Further, children's RSA seems to be related to behavioral synchrony in parent-child interaction; when there are fewer instances of behavioral and emotional synchrony, basal RSA tends to be lower in infants, indicating reduced physiological regulation (Feldman \& Eidelman, 2007). Thus, adaptive coregulation through the time- 
dependent coordination of parent and child physiological and behavioral states provides support for the development of the children's self-regulation of physiological responses.

Although parents and children tend to demonstrate synchrony in behavioral and physiological states in general, individual parent factors seem to play a crucial role in shaping parent-child coregulatory patterns. Parents who engage in either excessive involvement or under-involvement with children may limit opportunities for positive interactions, which in turn may impair coordination in physiological states (Feldman, 2012). For example, research has linked maternal sensitivity, or the appropriate involvement and responsiveness to children's needs, to synchrony in mothers' and children's physiological responses to stress. One study found that mothers' and children's cortisol responses were coordinated with one another when mothers were described as sensitive; however, this association was weaker for less sensitive mothers (Ruttle, Serbin, Stack, Schwartzman, \& Shirtcliff, 2011). Likewise, another study found synchrony in mother-child physiological states at higher levels of maternal sensitivity, however, no significant coordination between mother and child adrenocortical response was observed at low levels of maternal sensitivity (Sethre-Hofstad, Stansbury, \& Rice, 2002).

Parents who have had a history of maltreatment of their children, including physical or emotional abuse and neglect, also demonstrate coregulatory patterns with their children that are divergent, or tend to move away from one another over time (Creaven, Skowron, Hughes, Howard, \& Loken, 2014). In addition, when parents are less able to regulate their own physiological responses, synchronous coregulation patterns that support successful child selfregulation may become weaker, or possibly divergent. For example, mothers who exhibited elevated heart rates showed less physiological synchrony with their children (Creaven et al., 2014), whereas mothers who showed RSA withdrawal during a stressful interaction displayed 
greater behavioral synchrony with children (Giuliano, Skowron, \& Berkman, 2015). Such results suggest that individual parent factors, such as physiological responding and behavioral engagement, are important in promoting synchrony in parent-child coregulation. However, this hypothesis has not been explicitly examined in parents and preschool-aged children, during the developmental period that is crucial for the internalization of children's self-regulation skills via repeated parent-child interactions. Thus, the primary aim of the current study is to examine whether the maternal factors of basal physiology, measured as RSA during a relatively low stress task, and behavioral engagement during a parent-child interaction are associated with the degree of coregulatory synchrony in RSA observed between mothers and their preschoolers.

In the preschool years, parental engagement continues to be important, however, it may begin to take different forms as children's developmental needs change. As children develop greater self-regulation skills, the type of support they require from parents shifts from actions such as joint attention to incorporating more autonomy-supportive behavior, such as teaching (Grolnick, Deci, \& Ryan, 1997). Children's increasing behavioral regulation over the course of the early childhood period (between the ages of 2 years and 5 years) predicts a decline in parents' use of simple directives and an increase in teaching (Landry, Smith, Swank, \& MillerLoncar, 2000). Parent teaching has been shown to provide support for children's autonomy, including cognitive and social independence, whereas directives appear to have a negative influence on autonomy development after the age of 3 years (Eisenberg et al., 2010). Although parents' RSA responses to stress have been linked with parental engagement with infants (e.g. Mills-Koonce et al., 2009), no research has yet examined whether teaching is similarly associated with RSA coregulation between parents and preschool-aged children. Thus, the current study investigated the relationship between parent-child physiological coregulation and 
maternal teaching, as a measure of parents' behavioral engagement with their 3- to 4-year-old children.

Dyadic coregulation may be best measured using analytic models that account for temporal fluctuations in one's own behavior, affect, or physiology. Dynamic time series analyses have an advantage over linear analyses in that they quantify the coordination of physiological states as they change over time, better capturing the coregulation construct. For example, Gottman and colleagues utilized coupled autoregressive time series models to determine that an individual's behavior can be predicted as a function of both their prior behavior and the behavior of their spouse (Gottman, Swanson, \& Swanson, 2002). Lunkenheimer and colleagues (2015) used a similar strategy to examine coregulation in parent and child RSA across time. Their models provided a measure of the association between child RSA at a given time point and parents' concurrent RSA, and vice versa, when controlling for each individual's own intraindividual variability in RSA. The researchers found that parent RSA at a given time point positively predicted their child's concurrent RSA, and vice versa. However, they also found evidence for disruption of synchrony in RSA based on individual differences in child dysregulation. Specifically, when children showed higher levels of externalizing symptoms, parent and child RSA tended to diverge across time. Given the utility of fine-grained temporal analyses in elucidating relationships between individual factors and coregulatory dynamics, the current study employed multi-level coupled autoregressive modeling to assess parent-child physiological states as they changed moment-to-moment and how patterns of coordination were related to differences in maternal RSA and behavioral engagement. 


\section{Current Study}

Although prior research has established a link between parents' behavioral and physiological states and children's developing self-regulation, the mechanisms that connect parent factors to child outcomes are not well understood. Parent-child coregulation may be one important mechanism through which parents' behavioral and physiological states impact children's developing regulatory skills. The current study investigated this idea by using multilevel coupled autoregressive models to examine whether parent-child coregulation of RSA was predicted by differences in maternal factors. Specifically, we examined whether higher levels of parent basal RSA, measured as higher average RSA during the baseline phase of a dyadic parent-child problem-solving task, was associated with greater synchrony in RSA between parents and their preschool-aged children. In addition, we examined whether motherchild coregulation of RSA was predicted by the level of maternal behavioral engagement during the same task. Specifically, we examined whether higher levels of maternal teaching (a measure of behavioral engagement particularly important in this developmental period) was associated with greater synchrony in mother-child coregulation of RSA and whether higher levels of maternal behavioral disengagement was associated with weaker patterns in mother-child coregulation of RSA. Patterns of coregulation in mother and child RSA were modeled in real time (second-by-second) over the course of 18 minutes of dyadic interaction. 


\section{METHODS}

\section{Participants}

The current sample was derived from a larger sample of 100 mother-child dyads participated in a study examining behavioral, emotional, and physiological regulation in parentchild interactions. Families were recruited through local preschools and businesses and through email listserves of agencies serving families with young children. Dyads were excluded from the study if mothers could not speak and/or write in English, if the child had developmental delays, or if either the mother or child had a heart condition that would interfere with the collection of heart rate data. Participants identified as $86 \%$ White, $8 \%$ Biracial, 3\% Asian, and 3\% "other" race; $10 \%$ were of Hispanic or Latino ethnicity. Median annual family income was $\$ 65,000$ and the average level of education attained by parents was a college degree. Mothers reported their marital status as $79 \%$ married, $7 \%$ cohabitating, $7 \%$ single, $5 \%$ separated or divorced, and $1 \%$ remarried. Due to wireless interference with physiological data collection equipment, only 47 dyads from the total sample of 100 had complete RSA data across all interaction tasks and were therefore retained as the final sample for the current investigation. Families with complete RSA data differed from the larger sample in that they had higher annual income, $t=2.32, p<.05$ and their children were older, $t=-2.16, p<.05$. However, families in the subsample did not differ on duration of maternal teaching behavior, $t=-.085$, n.s., or maternal behavioral disengagement, $t=$ $-1.01, n . s .$, throughout the course of the interaction tasks.

\section{Procedure}

Participation in the study occurred over two time points; Time 1 occurred when children were approximately 3.5 years of age, and Time 2 occurred six months later, when children were 
approximately 4 years old. Data for the current investigation were taken from the Time 1 laboratory sessions. Mothers and children came to the laboratory for a 2.5 hour session where they participated in several dyadic interaction tasks. Children also participated in activities to measure self-regulation, effortful control, fear reactivity, and persistence. Mothers filled out surveys to provide information on family demographics, life stress, parent mental health, parenting practices, and child behavior and temperament. Heart rate and respiratory data for mothers and children were collected throughout the dyadic interaction tasks. All tasks were videotaped with the mother's consent. Participants were compensated for their time. All procedures were approved by the Institutional Review Board at Colorado State University.

Dyadic interaction tasks. Mothers and children completed several dyadic interaction tasks for a total of 18 minutes. First, mothers and children were engaged in a free play task for seven minutes. An experimenter placed toys out for mothers and children in a private room and they were instructed to "behave as you normally would at home." Next, mothers and children were engaged in a clean-up task for four minutes. After the free play period, the experimenter returned to the room after with a large bin and instructed the mother to have the child put the toys back in the bin. Mothers were asked to guide children using only their verbal instruction and were told not to physically aid children in putting the toys away.

Finally, mothers and children engaged in the Parent Child Challenge Task (PCCT; Lunkenheimer, Kemp, Lucas-Thompson, Cole, \& Albrecht, 2016), a teaching task designed to prompt the parent to guide the child to complete a 3D puzzle. Each dyad was given a puzzle toy that could be disassembled and reassembled in many different ways. Mothers were provided a guidebook and asked to help their children complete three particular puzzles; they were asked to use only their words and not to physically assist children to build the puzzle. The mother and 
child were informed that the child could earn a prize if all three puzzles were completed. In the baseline phase, mother and child were given four minutes to work together on the puzzle. In the stressor phase, the experimenter returned to the room and introduced a new time limit, informing the mother that they only had two minutes to finish the task. The dyad was actually allowed an additional three minutes to work on the puzzles. In the repair phase, the experimenter returned to the room with a prize for the child regardless of whether the puzzles were completed. The dyad was left alone for another three minutes to play with the prize together.

\section{Measures}

Respiratory sinus arrhythmia. Electrocardiogram (ECG) data was collected from mothers and children using three electrodes placed at the right clavicle and below the ribcage on the left (recording electrodes), and below the right ribcage (grounding electrode). Respiration was measured using a crystal respiratory belt. ECG and respiratory data were collected using handheld computers that were carried by the participants in backpacks and connected wirelessly to a desktop computer in the observation room. Interbeat interval data was processed and cleaned offline by a trained research assistant using Mindware Heart Rate Variability 3.0.13 software. Epochs requiring more than $10 \%$ editing due to artifact were dropped from the analysis. RSA magnitude was calculated as the natural logarithm of the variance of heart period within the frequency related to respiration $(0.24-1.04 \mathrm{~Hz}$ for children and $0.12-0.40 \mathrm{~Hz}$ for adults; Fracasso, Porges, Lamb, \& Rosenberg, 1994) using Biolab 2.5 software (Mindware Technologies, Gahanna, OH). Mean RSA was calculated for each 30 second epoch throughout the 18 minutes of dyadic interaction, for a total of 36 epochs. Due to wireless interference, only 47 families out of 100 in the larger study had complete and valid RSA data for all dyadic tasks. Mother basal RSA was measured as the average mother RSA across the baseline phase of the 
PCCT, which was prior to implementing the time constraint stressor, and thus represented a relatively low-stress period.

Maternal teaching and behavioral disengagement. Maternal behavior during the dyadic interaction tasks was coded offline by trained graduate and undergraduate research assistants according to the Dyadic Interaction Coding System, version 2.0 (Lunkenheimer, 2009) using Noldus Observer XT software, version 8.0. Behavior was classified moment-to-moment as: proactive structure - attempts to prompt, guide, or encourage desired behavior in the child; positive reinforcement - verbal expressions of reward, praise, or support of the child; emotional support - verbal and nonverbal expressions of endearment or empathy for the child; teaching offering explanation about how something works or clear instruction about a game or task; directive - clear and firm commands for behavior change; engagement - watching the child or interacting with the child socially; disengagement - lack of interaction with child, including ignoring child's bids for attention; intrusion - taking over the child's play or task-related behavior; or negative discipline - using criticism or threats to coerce the child to engage in desired behavior. All behaviors were mutually-exclusive, meaning that only one behavior was coded at a time and could not overlap.

Maternal teaching was selected as an index of active maternal engagement with the child based on prior research indicating the importance of parental autonomy-supportive behavior in early childhood for child outcomes (Grolnick et al., 1997). In addition, maternal disengagement was selected to reflect poorer or lower levels of social engagement with the child, which may be an important correlate of RSA regulation (Porges, 2007). Each of the behavioral variables was measured as the total duration in minutes that a mother exhibited the behavior throughout the full 18 minutes of dyadic interaction tasks. 


\section{Data Analytic Plan}

Mother and child coregulation of RSA and prediction of coregulation by each of the three separate maternal factors (basal RSA, teaching, and disengagement) were estimated using multilevel coupled autoregressive models fit in MPlus version 7.2 (Muthen \& Muthen, 19982012). Two separate models were run for each maternal factor, one predicting mother RSA and one predicting child RSA, in order to determine differential effects of maternal factors on coregulation for mothers and for children. The Level 1 within-dyad equations are displayed below.

$$
\begin{aligned}
& m R S A_{\mathrm{i}, \mathrm{t}}=\mu_{m i}+\beta_{m, \text { self } 1} m R S A_{i, t-1}+\beta_{m, \text { self } 2} m R S A_{i, t-2}+\beta_{m, \text { self } 3} m R S A_{i, t-3}+\beta_{m, c o} c R S A_{i, t}+\varepsilon_{m i, t} \\
& c R S A_{\mathrm{i}, \mathrm{t}}=\mu_{c i}+\beta_{c, \text { self } 1} c R S A_{i, t-1}+\beta_{c, \text { self } 2} c R S A_{i, t-2}+\beta_{c, \text { self } 3} c R S A_{i, t-3}+\beta_{c, \text { co }} m R S A_{i, t}+\varepsilon_{c i, t}
\end{aligned}
$$

In these equations, $m R S A_{\mathrm{i}, \mathrm{t}}$ and $c R S A_{\mathrm{i}, \mathrm{t}}$ represent the $i^{\text {th }}$ mother's and $i^{\text {th }}$ child's RSA at epoch $t$, respectively. Overall mean RSA, denoted as $\mu_{m i}$ for mothers and $\mu_{i i}$ for children, was included in the models to account for the effects that individual differences in mean RSA across all tasks might have on coregulation. Fixed effects for mothers' and children's own prior RSA at three

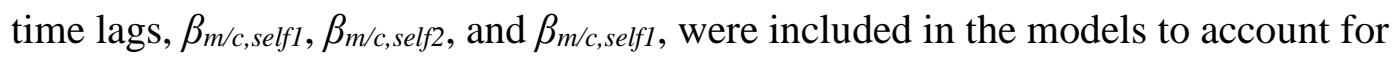
intraindividual variability in each individual's own fluctuations in RSA over time. Finally, the effect of the partner's concurrent RSA, $\beta_{m, c o}$ and $\beta_{c, c o}$, on mother or child RSA were estimated as a measure of coregulation between mothers and children. As these models incorporated RSA data from all 36 epochs of time for each mother and child, power to detect associations between variables was greater than if data were averaged across the interaction (Maxwell \& Delaney, 1990).

Next, we examined whether each of the three maternal factors (basal RSA, teaching, and disengagement) predicted the coregulation of mother and child RSA. Each of the maternal 
factors was entered as a Level 2 between-subjects predictor in separate models. The set of Level 2 between-subjects equations for mothers' basal RSA $\left(\alpha_{m}\right)$ predicting average fixed effects of mean maternal RSA $\left(\mu_{m i}\right)$, intraindividual variability in maternal RSA $\left(\beta_{m}\right.$,selfl, $\beta_{m}$, self 2 , and $\left.\beta_{m, \text { self } 3}\right)$, and mother's coregulation with child RSA $\left(\beta_{m, c o}\right)$ on mother RSA are displayed below. Note that fixed effects denoted as $\beta_{m / c}$ in the Level 1 equations are denoted as $\gamma_{m / c}$ in the Level 2 equations.

$$
\begin{gathered}
\mu_{m i}=\mu_{m i}+\gamma_{m}+\alpha_{m, \text { Mean Maternal Basal RSA }} \\
\beta_{m, \text { self } 1}=\gamma_{m, \text { self } 1}+\alpha_{m, \text { self } 1 \text { Maternal Basal RSA }} \\
\beta_{m, \text { self } 2}=\gamma_{m, \text { self } 2}+\alpha_{m, \text { self } 2 \text { Maternal Basal RSA }} \\
\beta_{m, \text { self } 3}=\gamma_{m, \text { self } 3}+\alpha_{m, \text { self } 3 \text { Maternal Basal RSA }} \\
\beta_{m, c o}=\gamma_{m, \text { co }}+\alpha_{c, \text { co Maternal Basal RSA }}
\end{gathered}
$$

The equivalent equations for mother basal RSA $\left(\alpha_{c}\right)$ predicting average fixed effects of mean

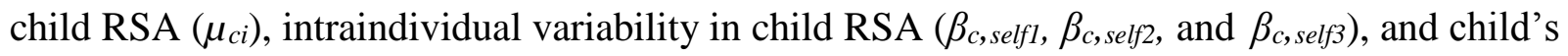
coregulation with mother RSA $\left(\beta_{c, c o}\right)$ on child RSA are displayed below.

$$
\begin{gathered}
\mu_{c i}=\mu_{c i}+\gamma_{c}+\alpha_{c, \text { Mean Maternal Basal RSA }} \\
\beta_{c, \text { self } 1}=\gamma_{c, \text { self } 1}+\alpha_{c, \text { self } 1 \text { Maternal Basal RSA }} \\
\beta_{c, \text { self } 2}=\gamma_{c, \text { self } 2}+\alpha_{c, \text { self } 2 \text { Maternal Basal RSA }} \\
\beta_{c, \text { self } 3}=\gamma_{c, \text { self } 3}+\alpha_{c, \text { self } 3 \text { Maternal Basal RSA }} \\
\beta_{c, c o}=\gamma_{c, c o}+\alpha_{m, \text { co Maternal Basal RSA }}
\end{gathered}
$$

Similar Level 2 equations were used to examine the effects of maternal teaching and maternal disengagement on mother-child RSA coregulation in separate models. Altogether, three sets of multi-level autoregressive models (six models total) were run in order to isolate the effect of each maternal factor on dyadic RSA coregulation. 


\section{RESULTS}

\section{Preliminary Analyses}

Several one-sample Kolmogorov-Smirnov tests were conducted to examine normality of level 2 predictors. The variables of maternal basal RSA, $M=6.13, S D=0.87, D(47)=0.12, p>.05$, and maternal teaching, $M=3.24, S D=1.33, D(46)=0.07, p>.05$, were both normally distributed. The variable of maternal disengagement was positively skewed, $M=0.46, S D=0.47, D(46)=0.22$, $p<.001$, and was therefore corrected by first adding a constant of 1 to each value, and then taking the natural log transformation of each value. All data presented for maternal disengagement reflect this log-transformed variable.

Mothers' mean RSA across the dyadic tasks $(M=6.12, S D=0.8)$ was not correlated with children's mean RSA $(M=5.3, S D=0.95, r=.15, p=.35)$. Maternal basal RSA in the PCCT was positively correlated with mothers' mean RSA across all three dyadic interaction tasks $(r=.93$, $p<.001)$. There was a marginal negative association between maternal teaching and maternal disengagement such that when mothers used more teaching behaviors, they tended to be less disengaged throughout the course of the dyadic task $(r=-.28, p=.06)$. We examined the relationship between key study variables and potential covariates, including SES, maternal education, and child age, given that these factors may have influenced mothers' and children's experience with dyadic problem-solving in the PCCT; none of these variables were significantly correlated with our primary study variables of interest and thus are not reported.

\section{Primary Analyses}

Maternal basal RSA. Results for the multilevel coupled autoregressive models with maternal basal RSA as the between-subjects (Level 2) predictor are displayed in Table 1. All 
intercepts were significant in the prediction of current RSA for both mothers and children. On average, both mothers and children showed significant increases in RSA throughout the course of the dyadic interaction, as indexed by significant and positive effects of prior RSA at 30-, 60-, and 90-second time lags in predicting individuals' current RSA. In addition, both mothers and children showed significant time-varying dependence with one another on average, as demonstrated by significant and positive effects of coregulation on current RSA in both the Table 1. Multilevel coupled autoregressive models predicting current RSA for mothers and children with maternal basal RSA as the between-subjects predictor.

\begin{tabular}{|c|c|c|c|c|c|}
\hline Mother RSA Model & Estimate & SE & Child RSA Model & Estimate & SE \\
\hline \multicolumn{3}{|l|}{ Fixed Effects: } & \multicolumn{3}{|l|}{ Fixed Effects: } \\
\hline Mother Mean RSA & $6.16^{* * *}$ & 0.04 & Child Mean RSA & $5.30^{* * *}$ & 0.08 \\
\hline Mother RSA (30-s lag) & $0.08^{* * *}$ & 0.03 & Child RSA (30-s lag) & $0.21^{* * *}$ & 0.04 \\
\hline Mother RSA (60-s lag) & $0.13^{* * *}$ & 0.03 & Child RSA (60-s lag) & $0.22^{* * *}$ & 0.05 \\
\hline Mother RSA (90-s lag) & $0.07^{*}$ & 0.03 & Child RSA (90-s lag) & $0.09^{*}$ & 0.04 \\
\hline Child Coregulation & $0.11^{* * *}$ & 0.03 & Mother Coregulation & $0.19^{* * *}$ & 0.00 \\
\hline $\begin{array}{l}\text { Maternal Basal RSA } \times \\
\text { Mother Mean RSA }\end{array}$ & $0.53^{* * *}$ & 0.05 & $\begin{array}{l}\text { Maternal Basal RSA } \times \\
\text { Child Mean RSA }\end{array}$ & $-0.14^{*}$ & 0.06 \\
\hline $\begin{array}{l}\text { Maternal Basal RSA } \times \\
\text { Mother RSA (30-s lag) }\end{array}$ & 0.04 & 0.03 & $\begin{array}{l}\text { Maternal Basal RSA } \times \\
\text { Child RSA (30-s lag) }\end{array}$ & 0.02 & 0.04 \\
\hline $\begin{array}{l}\text { Maternal Basal RSA } \times \\
\text { Mother RSA (60-s lag) }\end{array}$ & -0.06 & 0.04 & $\begin{array}{l}\text { Maternal Basal RSA } \times \\
\text { Child RSA (60-s lag) }\end{array}$ & -0.04 & 0.05 \\
\hline $\begin{array}{l}\text { Maternal Basal RSA } \times \\
\text { Mother RSA (90-s lag) }\end{array}$ & 0.00 & 0.03 & $\begin{array}{l}\text { Maternal Basal RSA } \times \\
\text { Child RSA (90-s lag) }\end{array}$ & 0.03 & 0.03 \\
\hline $\begin{array}{l}\text { Maternal Basal RSA } \times \\
\text { Child Coregulation }\end{array}$ & $-0.07^{*}$ & 0.03 & $\begin{array}{l}\text { Maternal Basal RSA } \times \\
\text { Mother Coregulation }\end{array}$ & -0.07 & 0.05 \\
\hline \multicolumn{3}{|c|}{ Random Variance Components: } & \multicolumn{3}{|c|}{ Random Variance Components: } \\
\hline Within Level Residual & $0.58^{* * *}$ & 0.03 & Within Level Residual & $0.80^{* * *}$ & 0.07 \\
\hline Between Level Residual & $0.02^{*}$ & 0.01 & Between Level Residual & $0.18^{*}$ & 0.09 \\
\hline
\end{tabular}

mother and child models. Specifically, mothers' RSA had a significant positive effect on children's concurrent RSA, above and beyond the effects of children's own mean and 
intraindividual variability in RSA, and likewise, children's RSA had a significant positive effect on mothers' concurrent RSA, above and beyond the effects of mothers' own mean and intraindividual variability in RSA. These results indicated synchrony, or time-dependent positive coordination, between mothers' and children's RSA, on average.

With respect to the between-subjects effects of maternal basal RSA, higher basal RSA in mothers was associated with higher overall mean maternal RSA across the interaction. Conversely, higher maternal basal RSA was associated with lower overall mean RSA in children across the interaction. With respect to coregulation, in the maternal model, a one-unit increase in mothers' basal RSA was associated with a -0.07 decrease in the effect of child RSA on concurrent mother RSA. Thus, at higher levels of maternal basal RSA, coordination between mothers' and children's RSA became weaker, and eventually divergent, as indicated by the estimated negative association between child RSA and mother RSA at a two-unit increase in mothers' basal RSA (estimated effect of child coregulation=-0.03) and above. Maternal basal RSA was not associated with intraindividual variability in RSA for either mother or child.

Maternal teaching. Results for the multilevel coupled autoregressive models with maternal teaching as the between-subjects predictor are displayed in Table 2. Similar to the prior model, both mothers and children demonstrated significant increases in RSA as well as timevarying dependence with one another on average, as indicated by significant intercepts for mean RSA, intraindividual variability in RSA, and RSA coregulation. With respect to the betweensubjects effects of maternal teaching, partner coregulation had a significantly larger effect on individuals' current RSA at higher levels of maternal teaching in both the mother and child models. In the mother model, the effect of child RSA on concurrent mother RSA was strengthened by an estimate of 0.05 for each additional minute that mothers spent using teaching 
behavior during the course of the interaction. Similarly, a one-minute increase in mothers' teaching behavior was associated with a 0.08 increase in the estimated positive effect of mother RSA on concurrent child RSA. Thus, a greater duration of maternal teaching predicted greater synchrony in mothers' and children's RSA. Maternal teaching was not associated with intraindividual variability in mothers' or children's RSA, or with the overall mean RSA for mother or child.

Table 2. Multilevel coupled autoregressive models predicting current RSA for mothers and children with maternal teaching as the between-subjects predictor.

\begin{tabular}{|c|c|c|c|c|c|}
\hline Mother RSA Model & Estimate & $\mathrm{SE}$ & Child RSA Model & Estimate & SE \\
\hline \multicolumn{3}{|l|}{ Fixed Effects: } & \multicolumn{3}{|l|}{ Fixed Effects: } \\
\hline Mother Mean RSA & $6.19^{* * *}$ & 0.08 & Child Mean RSA & $5.30^{* * *}$ & 0.07 \\
\hline Mother RSA (30-s lag) & $0.09^{* * *}$ & 0.03 & Child RSA (30-s lag) & $0.20^{* * * *}$ & 0.04 \\
\hline Mother RSA (60-s lag) & $0.15^{* * *}$ & 0.03 & Child RSA (60-s lag) & $0.23^{* * *}$ & 0.05 \\
\hline Mother RSA (90-s lag) & $0.06^{*}$ & 0.03 & Child RSA (90-s lag) & $0.09^{* *}$ & 0.04 \\
\hline Child Coregulation & $0.14^{* * *}$ & 0.03 & Mother Coregulation & $0.16^{* * *}$ & 0.03 \\
\hline $\begin{array}{r}\text { Maternal Teaching } \times \\
\text { Mother Mean RSA }\end{array}$ & -0.07 & 0.06 & $\begin{array}{l}\text { Maternal Teaching } \times \\
\text { Child Mean RSA }\end{array}$ & 0.01 & 0.06 \\
\hline $\begin{array}{l}\text { Maternal Teaching } \times \\
\text { Mother RSA (30-s lag) }\end{array}$ & 0.02 & 0.02 & $\begin{array}{l}\text { Maternal Teaching } \times \\
\text { Child RSA (30-s lag) }\end{array}$ & 0.02 & 0.03 \\
\hline $\begin{array}{l}\text { Maternal Teaching } \times \\
\text { Mother RSA (60-s lag) }\end{array}$ & -0.03 & 0.03 & $\begin{array}{l}\text { Maternal Teaching } \times \\
\text { Child RSA (60-s lag) }\end{array}$ & -0.04 & 0.03 \\
\hline $\begin{array}{l}\text { Maternal Teaching } \times \\
\text { Mother RSA (90-s lag) }\end{array}$ & 0.00 & 0.03 & $\begin{array}{l}\text { Maternal Teaching } \times \\
\text { Child RSA (90-s lag) }\end{array}$ & 0.00 & 0.03 \\
\hline $\begin{array}{l}\text { Maternal Teaching } \times \\
\text { Child Coregulation }\end{array}$ & $0.05^{* * *}$ & 0.02 & $\begin{array}{l}\text { Maternal Teaching } \times \\
\text { Mother Coregulation }\end{array}$ & $0.08^{* *}$ & 0.03 \\
\hline \multicolumn{3}{|c|}{ Random Variance Components: } & \multicolumn{3}{|c|}{ Random Variance Components: } \\
\hline Within Level Residual & $0.58^{* * *}$ & 0.03 & Within Level Residual & $0.80^{* * *}$ & 0.07 \\
\hline Between Level Residual & $0.25^{* * *}$ & 0.07 & Between Level Residual & $0.20^{*}$ & 0.09 \\
\hline
\end{tabular}

Maternal disengagement. Results for the multilevel coupled autoregressive models with maternal disengagement as the between-subjects predictor are displayed in Table 3. As in the prior two models, all of the intercepts were significant for both the mother and child models, 
indicating significant intraindividual increases in RSA and significant mother-child coregulation of RSA for dyads on average. With respect to the between-subjects effects of maternal disengagement, in the mother model, greater maternal disengagement was associated with

Table 3. Multilevel coupled autoregressive models predicting current RSA for mothers and children with maternal disengagement as the between-subjects predictor.

\begin{tabular}{|c|c|c|c|c|c|}
\hline Mother RSA Model & Estimate & SE & Child RSA Model & Estimate & SE \\
\hline \multicolumn{3}{|l|}{ Fixed Effects: } & \multicolumn{3}{|l|}{ Fixed Effects: } \\
\hline Mother Mean RSA & $6.18^{* * * *}$ & 0.08 & Child Mean RSA & $5.30^{* * * *}$ & 0.08 \\
\hline Mother RSA (30-s lag) & $0.09^{* * * *}$ & 0.03 & Child RSA (30-s lag) & $0.21^{* * * *}$ & 0.04 \\
\hline Mother RSA (60-s lag) & $0.15^{* * *}$ & 0.03 & Child RSA (60-s lag) & $0.22^{* * * *}$ & 0.05 \\
\hline Mother RSA (90-s lag) & $0.06^{*}$ & 0.03 & Child RSA (90-s lag) & $0.09^{*}$ & 0.04 \\
\hline Child Coregulation & $0.15^{* * * *}$ & 0.03 & Mother Coregulation & $0.19^{* * * *}$ & 0.00 \\
\hline $\begin{array}{l}\text { Maternal Disengagement } \times \\
\text { Mother Mean RSA }\end{array}$ & $-0.28^{\wedge}$ & 0.15 & $\begin{array}{l}\text { Maternal Disengagement } \times \\
\text { Child Mean RSA }\end{array}$ & 0.12 & 0.15 \\
\hline $\begin{array}{l}\text { Maternal Disengagement } \times \\
\text { Mother RSA (30-s lag) }\end{array}$ & -0.09 & 0.04 & $\begin{array}{l}\text { Maternal Disengagement } \times \\
\text { Child RSA (30-s lag) }\end{array}$ & $-0.16^{*}$ & 0.04 \\
\hline $\begin{array}{l}\text { Maternal Disengagement } \times \\
\text { Mother RSA (60-s lag) }\end{array}$ & 0.11 & 0.08 & $\begin{array}{l}\text { Maternal Disengagement } \times \\
\text { Child RSA (60-s lag) }\end{array}$ & 0.12 & 0.09 \\
\hline $\begin{array}{l}\text { Maternal Disengagement } \times \\
\text { Mother RSA }(90-\mathrm{s} \text { lag) }\end{array}$ & -0.09 & 0.05 & $\begin{array}{l}\text { Maternal Disengagement } \times \\
\text { Child RSA (90-s lag) }\end{array}$ & -0.05 & 0.52 \\
\hline $\begin{array}{l}\text { Maternal Disengagement } \times \\
\text { Child Coregulation }\end{array}$ & $-0.11^{*}$ & 0.05 & $\begin{array}{l}\text { Maternal Disengagement } \times \\
\text { Mother Coregulation }\end{array}$ & $-0.11^{\wedge}$ & 0.05 \\
\hline \multicolumn{3}{|c|}{ Random Variance Components: } & \multicolumn{3}{|c|}{ Random Variance Components: } \\
\hline Within Level Residual & $0.58^{* * * *}$ & 0.03 & Within Level Residual & $0.80^{* * *}$ & 0.06 \\
\hline Between Level Residual & $0.24^{* * *}$ & 0.07 & Between Level Residual & $0.16^{\wedge}$ & 0.08 \\
\hline
\end{tabular}

significant decreases in the effect of child RSA on mothers' current RSA, indicating weakened coregulation. Specifically, the effect of coregulation was reduced by -0.11 for every additional minute that mothers displayed behavioral disengagement during the course of the interaction, resulting in a negative association between child and mother RSA at higher levels of disengagement. In addition, there was a marginally significant association between maternal 
disengagement and mothers' overall mean RSA such that mothers who were more disengaged during the interaction had lower overall mean RSA.

With respect to the child model, greater maternal disengagement was related to decreases in children's RSA over time; specifically children's RSA at a 30-second lag negatively predicted children's current RSA. This declining RSA within child over time may have reflected children's greater physiological self-regulation when mothers were more disengaged during the interaction. Results for the effect of disengagement on coregulation in the child model followed a similar pattern as in the mother model, wherein the relationship between mothers' and children's RSA was weakened at higher levels of mother disengagement; however, this effect was only marginally significant in the child model. 


\section{DISCUSSION}

Although we know that parenting and parental stress physiology play an important role in parent-child interaction patterns, we have considerably more to learn about how these factors impact coregulation of physiology between parents and preschoolers. The purpose of the current study was to build upon this understanding through an examination of the coregulatory dynamics of parents' and children's RSA as they interacted with one another over the course of several dyadic tasks. We examined the association between several maternal factors and parent-child coregulation of RSA, including mothers' basal RSA, maternal behavioral engagement in the form of teaching, and maternal behavioral disengagement. We found that whereas increased duration of maternal teaching was related to greater time-dependent positive coordination in mother-child RSA over time, increased maternal disengagement was associated with weaker coordination and divergent (negative) coregulation at higher levels of disengagement.

Heightened maternal basal RSA was also related to divergent coregulatory patterns in RSA over time. To our knowledge, this is the first study to explore such associations among parents and preschool-aged children, although prior research and theory suggest a role for coregulation as a mechanism through which parents influence children's developing self-regulation (Calkins, 2011; Lunkenheimer et al., 2011). This work builds on prior research supporting that parents' physiological states underlie and support positive engagement with children (Ham \& Tronick, 2006; Mills-Koonce et al., 2009), which in turn provides support for children's physiological regulation (Conradt \& Ablow, 2010; Moore et al., 2009) through improved parent-child coregulation. 
In general, mothers and children showed synchrony in RSA throughout the course of the dyadic interaction; a finding that held across all six models, and corroborates prior research establishing and supporting physiological coregulation in parents and children (Creaven et al., 2014; Lunkenheimer et al., 2015; Ruttle et al., 2011). However, patterns of RSA coregulation appeared to vary in relation to individual maternal factors, such as the level of engagement mothers exhibited with their children in an active teaching task, as well as their physiological state during the course of the interaction. These findings are in line with prior work that has demonstrated that synchrony in mothers' and children's physiological states varies with regard to maternal sensitivity to children's needs (Ruttle et al., 2011; Sethre-Hofstad et al., 2002).

With regards to maternal teaching, coregulation in mothers' and children's RSA was greater when mothers spent more time using teaching behaviors with children, such as offering verbal instruction about the task or demonstrating how the puzzle worked. This finding was true in both the mother and child models, and after accounting for intraindividual variability in RSA over time, indicating a robust effect. This type of teaching behavior has been shown to support children's autonomy across the early childhood period (Eisenberg et al., 2010; Landry et al., 2000). In the present study, teaching behavior, particularly in the context of the challenging interactional task that required the guidance of parents, represented appropriate parental engagement that reflected responsiveness to children's developmental needs. Prior research has shown that parents' responsiveness to children's needs is related to improvements in RSA regulation during infancy (Conradt \& Ablow, 2010; Moore et al., 2009). Moreover, children seem better able to regulate RSA when working with their parents versus working alone (Calkins et al., 2008). The finding that increased behavioral engagement in the form of teaching is also related to parent-child RSA coregulation may point to a potential explanatory mechanism for the 
way in which parent engagement supports children's regulation of RSA in early childhood. It is possible that task-appropriate teaching behavior also facilitated increased behavioral and emotional synchrony between mothers and children, which in turn may have promoted physiological coregulation (Feldman, 2012). Future studies relating behavioral and physiological coregulatory patterns in parent-child interactions could further help to elucidate the mechanisms connecting parent engagement and the self-regulation and coregulation of parasympathetic processes.

Maternal disengagement, which encompassed behaviors such as the mother working alone and ignoring children's bids for attention, produced an opposite pattern from that of teaching and was associated with divergent coregulatory patterns, in which mothers' and children's RSA were negatively associated at higher levels of mother disengagement. This finding can be interpreted in light of theory that suggests that mothers and children develop synchrony in physiological states through engaging in mutually responsive behavioral exchanges (Feldman, 2012). If parents ignore children's bids for engagement, or are otherwise preoccupied and not available for such exchanges, parent-child physiological coregulation may be weakened. Additionally, greater maternal disengagement was associated with children's declining RSA over the course of the interaction. This finding may support prior work that suggests a link between lower parental involvement with children and children's RSA dysregulation (Feldman \& Eidelman, 2007). Porges' Polyvagal theory suggests that higher RSA during relatively lowstress periods facilitates interpersonal engagement (Porges, 2007). In line with this theory, mothers in this sample who were disengaged during longer periods also tended to have lower RSA throughout the dyadic interaction, however, this effect was of marginal significance. 
Contrary to expectation, higher maternal basal RSA was associated with divergent coregulatory patterns in mothers' and children's RSA. Maintaining a high level of RSA during relatively low-stress periods is thought to reflect increased activity of the "vagal brake," slowing heart rate and facilitating engagement with the environment (Beauchaine, 2001; Porges, 2007). Based on Porges' theory and prior research findings (Ham \& Tronick, 2006; Mills-Koonce et al., 2009; Porges, 2007), it was expected that higher RSA would have facilitated mothers' behavioral engagement with their children during the course of the dyadic interaction, therefore promoting physiological synchrony (Feldman, 2012). However, in our case, basal RSA was measured across the basal phase of a teaching task, in which mothers were expected to actively guide children in building challenging puzzles. Although the baseline condition of this task constituted a relatively low-stress period compared to the challenge condition, it still required some effort from mothers. Thus, higher-than-average RSA in this context might have reflected a lack of withdrawal of RSA in response to the cognitive and emotional challenge of assisting and teaching their children, which could have been related to a reduced regulatory response or effort on the part of mothers (Porges, 2007). The fact that higher mother basal RSA was also related to lower mean child RSA supports this explanation, in that lower child RSA could reflect that children were expending greater effort, or experiencing greater distress throughout the dyadic interaction. In future studies, the incorporation of a separate condition in which to measure resting RSA and the examination of repeated dyadic measures over time will help to verify this relationship between parent basal RSA and parent-child coregulation of RSA.

The use of multilevel autoregressive models was a strength of the study in that this analytic technique accounted for intraindividual fluctuations in RSA over time. This finegrained assessment also allowed for investigation of the coordination of mothers' and children's 
physiological states as they unfolded over time, which better captured the dynamic coregulation construct. In addition, whereas much of the research on parent-child coregulatory patterns focuses on infancy, there are relatively fewer investigations of how such patterns persist into early childhood and beyond. Thus, findings from the current study contribute novel information that builds upon and extends prior work on the relational contexts in which children's regulatory skills develop.

Nevertheless, findings should be interpreted in light of study limitations. First, as relationships were examined cross-sectionally, we cannot determine whether maternal factors led to changes in RSA coregulation, or vice versa. We interpreted observed associations in light of theory and prior work, but given that the relationships were not causal, additional explanations of the data may also be plausible. For example, it is possible that mothers with higher basal RSA had children who were more proactive during the challenge task and required less parental scaffolding, which might explain the observed divergence in mother and child RSA over time when mothers had higher basal RSA. In addition, we measured basal RSA as the average RSA throughout a relatively low-stress period, however, this is not always how RSA is defined and measured across empirical studies. For example, in some prior studies, what we refer to as basal RSA has been operationalized as "resting RSA," and measured across a resting period where parents and children were not engaged in interaction, or task demands were minimal. Qualitative differences in measurement of basal or resting RSA across parent-child studies may be one reason why current findings regarding maternal basal RSA were somewhat inconsistent with what may be expected based on prior research. Future studies may benefit from including a true resting period from which to analyze parents' RSA. Finally, our sample consisted of relatively homogeneous families who were mostly white, married, and college-educated on average and 
included only mother-child dyads. Thus, findings may not extend to more socioeconomically diverse families or apply to interactions between fathers and children. Prior research has shown that maternal engagement tends to adopt a social focus with an emphasis on the mother-child interaction, paternal engagement typically involves a more triadic orientation with greater focus on the external environment, encouraging exploration (Feldman, 2007). Future research should examine whether the unique quality and dynamics of paternal engagement impacts parent-child physiological coregulation differently than maternal engagement.

The period of early childhood is an important stage for the development of selfregulatory competence, however, children still rely on scaffolding from parents to reach important milestones of self-regulatory development (Diamond \& Aspinwall, 2003). Current findings suggest that mothers' behavioral engagement with children and basal physiological state during parent-child interactions are related to their degree of coregulation of RSA, which may serve as a mechanism through which parents support children's burgeoning self-regulation (Calkins, 2011). The relationship between parental engagement and physiological coregulation with children established in the current study suggests that the developmentally-appropriate scaffolding of children not only directly supports their developing autonomy but also promotes the positive dyadic coregulatory processes that facilitate children's self-regulation skills. 


\section{REFERENCES}

Beauchaine, T. P. (2001). Vagal tone, development, and Gray's motivational theory: Toward an integrated model of autonomic nervous system functioning in psychopathology. Development and Psychopathology, 13(2), 183-214. doi: 10.1017/S0954579401002012

Berntson, G. G., Cacioppo, J. T., \& Quigley, K. S. (1993). Respiratory sinus arrhythmia: Autonomic origins, physiological mechanisms, and psychophysiological implications. Psychophysiology, 30(2), 183-196. doi: 10.1111/j.1469-8986.1993.tb01731.x

Blandon, A. Y., Calkins, S. D., Keane, S. P., \& O'Brien, M. (2010). Contributions of child's physiology and maternal behavior to children's trajectories of temperamental reactivity. Developmental Psychology, 46(5), 1089-1102. doi: 10.1037/a0020678

Bornstein, M. H., \& Suess, P. E. (2000). Child and mother cardiac vagal tone: Continuity, stability, and concordance across the first 5 years. Developmental Psychology, 36(1), 5465. doi: 10.1037/0012-1649.36.1.54

Brooker, R. J., Buss, K. A., Lemery-Chalfant, K., Aksan, N., Davidson, R. J., \& Goldsmith, H. H. (2013). The development of stranger fear in infancy and toddlerhood: Normative development, individual differences, antecedents, and outcomes. Developmental Science, 16(6), 864-878.

Calkins, S. D. (2011). Caregiving as coregulation: Psychobiological processes and child functioning. In A. Booth, S. M. McHale, N. S. Landale, A. Booth, S. M. McHale \& N. S. Landale (Eds.), Biosocial foundations of family processes. (pp. 49-59). New York, NY, US: Springer Science + Business Media. 
Calkins, S. D., \& Dedmon, S. E. (2000). Physiological and behavioral regulation in two-year-old children with aggressive/destructive behavior problems. Journal of Abnormal Child Psychology, 28(2), 103-118. doi: 10.1023/A:1005112912906

Calkins, S. D., Graziano, P. A., Berdan, L. E., Keane, S. P., \& Degnan, K. A. (2008). Predicting cardiac vagal regulation in early childhood from maternal-child relationship quality during toddlerhood. Developmental Psychobiology, 50(8), 751-766. doi:

10.1002/dev.20344

Calkins, S. D., Graziano, P. A., \& Keane, S. P. (2007). Cardiac vagal regulation differentiates among children at risk for behavior problems. Biological Psychology, 74(2), 144-153. doi: 10.1016/j.biopsycho.2006.09.005

Calkins, S. D., \& Keane, S. P. (2004). Cardiac Vagal Regulation across the Preschool Period: Stability, Continuity, and Implications for Childhood Adjustment. Developmental Psychobiology, 45(3), 101-112. doi: 10.1002/dev.20020

Calkins, S. D., Smith, C. L., Gill, K. L., \& Johnson, M. C. (1998). Maternal interactive style across contexts: Relations to emotional, behavioral, and physiological regulation during toddlerhood. Social Development, 7(3), 350-369. doi: 10.1111/1467-9507.00072

Cole, P. M., Martin, S. E., \& Dennis, T. A. (2004). Emotion Regulation as a Scientific Construct: Methodological Challenges and Directions for Child Development Research. Child Development, 75(2), 317-333. doi: 10.1111/j.1467-8624.2004.00673.x

Conradt, E., \& Ablow, J. (2010). Infant physiological response to the still-face paradigm: Contributions of maternal sensitivity and infants' early regulatory behavior. Infant Behavior \& Development, 33(3), 251-265. doi: 10.1016/j.infbeh.2010.01.001 
Creaven, A. M., Skowron, E. A., Hughes, B. M., Howard, S., \& Loken, E. (2014). Dyadic concordance in mother and preschooler resting cardiovascular function varies by risk status. Developmental Psychobiology, 56(1), 142-152. doi: 10.1002/dev.21098

Dale, L. P., O'Hara, E. A., Schein, R., Inserra, L., Keen, J., Flores, M., \& Porges, S. W. (2011). Measures of infant behavioral and physiological state regulation predict 54-month behavior problems. Infant Mental Health Journal, 32(4), 473-486. doi: 10.1002/imhj.20306

Diamond, L. M., \& Aspinwall, L. G. (2003). Emotion regulation across the life span: An integrative perspective emphasizing self-regulation, positive affect, and dyadic processes. Motivation and Emotion, 27(2), 125-156. doi: 10.1023/A:1024521920068

Doussard-Roosevelt, J. A., Montgomery, L. A., \& Porges, S. W. (2003). Short-term stability of physiological measures in kindergarten children: Respiratory sinus arrhythmia, heart period, and cortisol. Developmental Psychobiology, 43(3), 231-242. doi:

10.1002/dev.10136

Ebisch, S. J., Aureli, T., Bafunno, D., Cardone, D., Romani, G. L., \& Merla, A. (2012). Mother and child in synchrony: Thermal facial imprints of autonomic contagion. Biological Psychology, 89(1), 123-129. doi: 10.1016/j.biopsycho.2011.09.018

Eisenberg, N., Vidmar, M., Spinrad, T. L., Eggum, N. D., Edwards, A., Gaertner, B., \& Kupfer, A. (2010). Mothers' teaching strategies and children's effortful control: A longitudinal study. Developmental Psychology, 46(5), 1294-1308. doi: 10.1037/a0020236

Feldman, R. (2007). Parent-infant synchrony and the construction of shared timing; Physiological precursors, developmental outcomes, and risk conditions. Journal of Child Psychology and Psychiatry, 48(3-4), 329-354. doi: 10.1111/j.1469-7610.2006.01701.x 
Feldman, R. (2012). Bio-behavioral synchrony: A model for integrating biological and microsocial behavioral processes in the study of parenting. Parenting: Science and Practice, 12(2-3), 154-164. doi: 10.1080/15295192.2012.683342

Feldman, R., \& Eidelman, A. I. (2007). Maternal postpartum behavior and the emergence of infant-mother and infant-father synchrony in preterm and full-term infants: the role of neonatal vagal tone. Dev Psychobiol, 49(3), 290-302. doi: 10.1002/dev.20220

Feldman, R., Magori-Cohen, R., Galili, G., Singer, M., \& Louzoun, Y. (2011). Mother and infant coordinate heart rhythms through episodes of interaction synchrony. Infant Behavior \& Development, 34(4), 569-577. doi: 10.1016/j.infbeh.2011.06.008

Fox, N. A. (1989). Psychophysiological correlates of emotional reactivity during the first year of life. Developmental Psychology, 25(3), 364-372. doi: 10.1037/0012-1649.25.3.364

Fracasso, M. P., Porges, S. W., Lamb, M. E., \& Rosenberg, A. A. (1994). Cardiac activity in infancy: Reliability and stability of individual differences. Infant Behavior \& Development, 17(3), 277-284. doi: 10.1016/0163-6383(94)90006-X

Giuliano, R. J., Skowron, E. A., \& Berkman, E. T. (2015). Growth models of dyadic synchrony and mother-child vagal tone in the context of parenting at-risk. Biological Psychology, 105, 29-36. doi: 10.1016/j.biopsycho.2014.12.009

Gottman, J., Swanson, C., \& Swanson, K. (2002). A general systems theory of marriage: Nonlinear difference equation modeling of marital interaction. Personality and social psychology review, 6(4), 326-340.

Graziano, P. A., \& Derefinko, K. (2013). Cardiac vagal control and children's adaptive functioning: A meta-analysis. Biological Psychology, 94(1), 22-37. doi: http://dx.doi.org/10.1016/j.biopsycho.2013.04.011 
Graziano, P. A., Keane, S. P., \& Calkins, S. D. (2007). Cardiac Vagal Regulation and Early Peer Status. Child Development, 78(1), 264-278. doi: 10.1111/j.1467-8624.2007.00996.x

Grolnick, W. S., Deci, E. L., \& Ryan, R. M. (1997). Internalization within the family: The selfdetermination theory perspective. In J. E. Grusec, L. Kuczynski, J. E. Grusec \& L. Kuczynski (Eds.), Parenting and children's internalization of values: A handbook of contemporary theory. (pp. 135-161). Hoboken, NJ, US: John Wiley \& Sons Inc.

Gunnar, M. R., Porter, F. L., Wolf, C. M., Rigatuso, J., \& Larson, M. C. (1995). Neonatal stress reactivity: Predictions to later emotional temperament. Child Development, 66(1), 1-13. doi: $10.2307 / 1131186$

Ham, J., \& Tronick, E. D. (2006). Infant Resilience to the Stress of the Still-Face. Annals of the New York Academy of Sciences, 1094(1), 297-302. doi: 10.1196/annals.1376.038

Hill-Soderlund, A. L., Mills-Koonce, W. R., Propper, C., Calkins, S. D., Granger, D. A., Moore, G. A., . . Cox, M. J. (2008). Parasympathetic and sympathetic responses to the strange situation in infants and mothers from avoidant and securely attached dyads. Developmental Psychobiology, 50(4), 361-376. doi: 10.1002/dev.20302

Huffman, L. C., Bryan, Y. E., del Carmen, R., Pedersen, F. A., Doussard-Roosevelt, J. A., \& Porges, S. W. (1998). Infant temperament and cardiac vagal tone: Assessments at twelve weeks of age. Child Development, 69(3), 624-635. doi: 10.2307/1132194

Kemp, A. H., Quintana, D. S., Gray, M. A., Felmingham, K. L., Brown, K., \& Gatt, J. M. (2010). Impact of depression and antidepressant treatment on heart rate variability: a review and meta-analysis. Biol Psychiatry, 67(11), 1067-1074. doi: 10.1016/j.biopsych.2009.12.012 
Kennedy, A. E., Rubin, K. H., Hastings, P. D., \& Maisel, B. (2004). Longitudinal Relations between Child Vagal Tone and Parenting Behavior: 2 to 4 Years. Developmental Psychobiology, 45(1), 10-21. doi: 10.1002/dev.20013

Kircanski, K., Waugh, C. E., Camacho, M. C., \& Gotlib, I. H. (2016). Aberrant parasympathetic stress responsivity in pure and co-occurring major depressive disorder and generalized anxiety disorder. Journal of Psychopathology and Behavioral Assessment, 38(1), 5-19. doi: $10.1007 / \mathrm{s} 10862-015-9493-\mathrm{y}$

Landry, S. H., Smith, K. E., Swank, P. R., \& Miller-Loncar, C. L. (2000). Early maternal and child influences on children's later independent cognitive and social functioning. Child Development, 71(2), 358-375. doi: 10.1111/1467-8624.00150

Lunkenheimer, E. S. (2009). Dyadic interaction coding manual. Unpublished manual. Colorado State University. Fort Collins, CO.

Lunkenheimer, E. S., Kemp, C. J., Lucas-Thompson, R. G., Cole, P. M., \& Albrecht, E. C. (2016). Assessing Biobehavioural Self-Regulation and Coregulation in Early Childhood: The Parent-Child Challenge Task. Infant and Child Development, n/a-n/a. doi: 10.1002/icd.1965

Lunkenheimer, E. S., Olson, S. L., Hollenstein, T., Sameroff, A. J., \& Winter, C. (2011). Dyadic flexibility and positive affect in parent-child coregulation and the development of child behavior problems. Development and Psychopathology, 23(2), 577-591. doi: 10.1017/S095457941100006X

Lunkenheimer, E. S., Tiberio, S. S., Buss, K. A., Lucas-Thompson, R. G., Boker, S. M., \& Timpe, Z. C. (2015). Coregulation of respiratory sinus arrhythmia between parents and 
preschoolers: Differences by children's externalizing problems. Developmental Psychobiology, 57(8), 994-1003. doi: 10.1002/dev.21323

Manini, B., Cardone, D., Ebisch, S. J. H., Bafunno, D., Aureli, T., \& Merla, A. (2013). Mom feels what her child feels: Thermal signatures of vicarious autonomic response while watching children in a stressful situation. Frontiers in Human Neuroscience, 7. doi: 10.3389/fnhum.2013.00299

Maxwell, S. E., \& Delaney, H. D. (1990). Designing experiments and analyzing data: A model comparison perspective. Belmont, CA, US: Wadsworth/Thomson Learning.

Miller, J. G., Chocol, C., Nuselovici, J. N., Utendale, W. T., Simard, M., \& Hastings, P. D. (2013). Children's dynamic RSA change during anger and its relations with parenting, temperament, and control of aggression. Biological Psychology, 92(2), 417-425. doi: 10.1016/j.biopsycho.2012.12.005

Mills-Koonce, W. R., Propper, C., Gariepy, J.-L., Barnett, M., Moore, G. A., Calkins, S., \& Cox, M. J. (2009). Psychophysiological correlates of parenting behavior in mothers of young children. Developmental Psychobiology, 51(8), 650-661. doi: 10.1002/dev.20400

Moore, G. A., Hill-Soderlund, A. L., Propper, C. B., Calkins, S. D., Mills-Koonce, W. R., \& Cox, M. J. (2009). Mother-infant vagal regulation in the face-to-face still-face paradigm is moderated by maternal sensitivity. Child Development, 80(1), 209-223. doi: 10.1111/j.1467-8624.2008.01255.x

Muthen, L. K., \& Muthen, B. O. (1998-2012). Mplus user's guide: Seventh edition. Los Angeles, CA: Muthen \& Muthen.

Olson, S. L., \& Lunkenheimer, E. S. (2009). Expanding concepts of self-regulation to social relationships: Transactional processes in the development of early behavioral adjustment. 
In A. Sameroff \& A. Sameroff (Eds.), The transactional model of development: How children and contexts shape each other. (pp. 55-76). Washington, DC, US: American Psychological Association.

Oppenheimer, J. E., Measelle, J. R., Laurent, H. K., \& Ablow, J. C. (2013). Mothers' vagal regulation during the Still-Face Paradigm: Normative reactivity and impact of depression symptoms. Infant Behavior \& Development, 36(2), 255-267. doi:

10.1016/j.infbeh.2013.01.003

Porges, S. W. (1991). Vagal tone: An autonomic mediator of affect. In J. Garber, K. A. Dodge, J. Garber \& K. A. Dodge (Eds.), The development of emotion regulation and dysregulation. (pp. 111-128). New York, NY, US: Cambridge University Press.

Porges, S. W. (2003). The Polyvagal Theory: Phylogenetic contributions to social behavior. Physiology \& Behavior, 79(3), 503-513. doi: 10.1016/S0031-9384(03)00156-2

Porges, S. W. (2007). The polyvagal perspective. Biological Psychology, 74(2), 116-143. doi: 10.1016/j.biopsycho.2006.06.009

Porges, S. W., Doussard-Roosevelt, J. A., \& Maita, A. K. (1994). Vagal tone and the physiological regulation of emotion. Monographs of the Society for Research in Child Development, 59(2-3), 167-186. doi: 10.2307/1166144

Porges, S. W., Doussard-Roosevelt, J. A., Portales, A. L., \& Greenspan, S. I. (1996). Infant regulation of the vagal 'brake' predicts child behavior problems: A psychobiological model of social behavior. Developmental Psychobiology, 29(8), 697-712. doi:

10.1002/(SICI)1098-2302(199612)29:8<697::AID-DEV5>3.0.CO;2-O 
Porges, S. W., Doussard-Roosevelt, J. A., Portales, L. A., \& Suess, P. E. (1994). Cardiac vagal tone: Stability and relation to difficultness in infants and 3-year-olds. Developmental Psychobiology, 27(5), 289-300. doi: 10.1002/dev.420270504

Posner, M. I., \& Rothbart, M. K. (2000). Developing mechanisms of self-regulation. Development and Psychopathology, 12(3), 427-441. doi: 10.1017/S0954579400003096

Ruttle, P. L., Serbin, L. A., Stack, D. M., Schwartzman, A. E., \& Shirtcliff, E. A. (2011). Adrenocortical attunement in mother-child dyads: Importance of situational and behavioral characteristics. Biological Psychology, 88(1), 104-111. doi:

10.1016/j.biopsycho.2011.06.014

Sameroff, A. J., \& MacKenzie, M. J. (2003). Research strategies for capturing transactional models of development: The limits of the possible. Development and Psychopathology, 15(3), 613-640. doi: 10.1017/S0954579403000312

Sethre-Hofstad, L., Stansbury, K., \& Rice, M. A. (2002). Attunement of maternal and child adrenocortical response to child challenge. Psychoneuroendocrinology, 27(6), 731-748. doi: 10.1016/S0306-4530(01)00077-4

Siegel, D. J. (2001). Toward an interpersonal neurobiology of the developing mind: Attachment relationships, 'mindsight,' and neural integration. Infant Mental Health Journal, 22(1-2), 67-94. doi: 10.1002/1097-0355(200101/04)22:1<67::AID-IMHJ3>3.0.CO;2-G

Stifter, C. A., \& Corey, J. M. (2001). Vagal regulation and observed social behavior in infancy. Social Development, 10(2), 189-201. doi: 10.1111/1467-9507.00158

Tronick, E. Z. (1989). Emotions and emotional communication in infants. American Psychologist, 44(2), 112-119. doi: 10.1037/0003-066X.44.2.112 
Wilson, B. J., \& Gottman, J. M. (1996). Attention-the shuttle between emotion and cognition: Risk, resiliency, and physiological bases. In E. M. Hetherington, E. A. Blechman, E. M. Hetherington \& E. A. Blechman (Eds.), Stress, coping, and resiliency in children and families. (pp. 189-228). Hillsdale, NJ, England: Lawrence Erlbaum Associates, Inc. 